\title{
Editorial: Transcriptional Regulation in Cancers and Metabolic Diseases
}

\author{
Wen Zhou ${ }^{1,2,3 *}$ \\ ${ }^{1}$ Department of Biological Sciences, Columbia University, New York, NY, USA, ${ }^{2}$ Braman Family Breast Cancer Institute, \\ University of Miami Sylvester Comprehensive Cancer Center, University of Miami Miller School of Medicine, Miami, FL, USA, \\ ${ }^{3}$ Molecular Oncology Research Program, Division of Surgical Oncology, Dewitt Daughtry Department of Surgery, University \\ of Miami Miller School of Medicine, Miami, FL, USA
}

Keywords: p53, nuclear receptor, cell cycle, cancer stem cell, epigenetics, microRNA, exosome, transcriptional regulation

I am privileged to edit this Research Topic, Transcriptional Regulation in Cancers and Metabolic Diseases under the guidance of Dr. Carol Prives. We hereby thank Drs. Antonino Belfiore and Claire Perks for giving us the opportunity of editing the Research Topic. Given the large number of recent publications on transcriptional regulation, this Research Topic is timely needed. It covers many research hotspots, including p53's gain-of-function mutation, p63's role in epithelial cells, mitosis- or senescence-related transcription, and cancer-specific exosome. Overall, this Research Topic reviews and updates the current trends in transcriptional regulation.

Two Mini Reviews summarize the roles of heat shock proteins in transcriptional regulation $(1,2)$. In the first Mini Review, Alexandrova and Marchenko focused on a heat shock protein HSF1 in mutant p53 (mutp53)-mediated oncogenic activation. They highlighted a novel connection between HSF1 and mutp53 and hypothesized that pharmaceutically disrupting HSF1-mutp53 cooperation might be beneficial to cancer patients. In the second Mini Review, Khurana and Bhattacharyya outlined mechanisms through which another heat shock protein HSP90 activates gene expression. That is, HSP90 co-activates transcription factors, interacts with chromatin remodeling factors, and evicts histones from certain gene promoters.

Three Reviews explain transcriptional regulation of fate-determining transcription factors or cofactors (3-5). In the first Review, Yoh and Prywes illustrated the regulatory network that impinges upon the key epithelial transcription factor p63. In the second Review, Yoo and colleagues focused on a ubiquitin-fold modifier, UFM1 in breast cancer. In the third Review, Sun and colleagues categorized lysine acetyltransferases in normal and abnormal development of blood cells. Notably, Sun et al. reported current drug developments in lysine acetyltransferase inhibitors.

Two Original research articles evaluate transcriptional regulation in uveal melanoma and aging, respectively $(6,7)$. In the first Original research article, Huffman, Carstens, and Martinez profiled the expression levels of 48 human NRs across a panel of cell lines from uveal melanoma, cutaneous melanoma and melanocytes. In addition, the NR-to-NR and NR-to-genome expression correlation analyses identified RXR $\gamma$ as a potential driver for melanoma-specific signaling, and ERR $\alpha$ as the uveal-melanoma-specific NR. In the second Original research article, Ma and colleagues analyzed microarray data of differentially expressed genes after the knockdown of the cellular senescenceinhibited gene (CSIG). CSIG, originally identified by this research group, is critical in regulating cell senescence, cell cycle progression, stress response, and tumor metastasis. In this article, they discovered novel CSIG targets that correlate with senescence. Interestingly, they inferred that CSIG regulates the stability of certain target-gene transcripts.

Nath and colleagues presented an interesting Review on mitotic proteins in cancer development (8). As the authors pointed out, deregulated transcriptional regulation of mitotic genes are common in cancers, yet mutations are rarely observed for mitotic genes. Cell cycle-related transcription program is interesting and warrants further investigation. 
One Review and one Opinion article appraised the possible use of exosome in early cancer diagnosis $(9,10)$. In the Review article, Qin and colleagues summarized the recent progress on the identification of miRNAs from tumor samples. They grouped different miRNAs based on their location as the cytosolic, bodyfluid or exosomal miRNAs. The authors proposed exosomal miRNAs as diagnostic biomarkers for lung tumors. In addition, they described the current methods for the isolation and detection of these RNAs in the tumor samples. Moreover, the authors hypothesized how miRNAs have been transported/released into body fluids from the tumor cells. In accompanying Opinion article, Oltra supported cancer-associated exosome in cancer diagnosis and prognosis.

In the end of this Research Topic, Davis and colleagues presented a very interesting Review on Nanotetrac in treating cancers $(11,12)$. In many cancers, Thyroid hormones, $\mathrm{T}_{3}$ and $\mathrm{T}_{4}$ have pro-angiogenic effects, which might be mediated by $\alpha_{V} \beta_{3}$

\section{REFERENCES}

1. Alexandrova EM, Marchenko ND. Mutant p53 - heat shock response oncogenic cooperation: a new mechanism of cancer cell survival. Front Endocrinol (2015) 6:1-7. doi:10.3389/fendo.2015.00053

2. Khurana N, Bhattacharyya S. Hsp90, the concertmaster: tuning transcription. Front Oncol (2015) 5:1-7. doi:10.3389/fonc.2015.00100

3. Yoh K, Prywes R. Pathway regulation of p63, a director of epithelial cell fate. Front Endocrinol (2015) 6:1-9. doi:10.3389/fendo.2015.00051

4. Yoo HM, Park JH, Jeon YJ, Chung CH. Ubiquitin-fold modifier 1 acts as a positive regulator of breast cancer. (2015) 6:1-7. doi:10.3389/fendo.2015.00036

5. Sun X-J, Man N, Tan Y, Nimer SD, Wang L. The role of histone acetyltransferases in normal and malignant hematopoiesis. Front Oncol (2015) 5:1-11. doi: $10.3389 /$ fonc. 2015.00108

6. Huffman KE, Carstens R, Martinez ED. A subset of nuclear receptors are uniquely expressed in uveal melanoma cells. Front Endocrinol (2015) 6:1-10. doi:10.3389/fendo.2015.00093

7. Ma L, Zhao W, Zhu F, Yuan F, Xie N, Li T, et al. Global characteristics of CSIGassociated gene expression changes in human HEK293 cells and the implications for CSIG regulating cell proliferation and senescence. Front Endocrinol (2015) 6:1-11. doi:10.3389/fendo.2015.00069

8. Nath S, Ghatak D, Das P, Roychoudhury S. Transcriptional control of mitosis: deregulation and cancer. Front Endocrinol (2015) 6:1-10. doi:10.3389/fendo. 2015.00060 integrin. Nanotetrac is a nanoparticulate preparation of a $\mathrm{T}_{4}$ substitute, tetrac. Nanotetrac blocks $T_{4}$-triggered $\alpha_{V} \beta_{3}$-mediated transcriptional regulation. Therefore, Nanotetrac might be useful in treating cancers.

By compiling all these excellent manuscripts into one Research Topic, we hope that our readers will find this Research Topic enlightening. We owe our thanks to the staff of Frontiers Endocrinology Office, for their work in the completion of this Research Topic. We are particularly thankful to Davor Vidic, Shaun Evans, Caroline Drage and Byron Bitanihirwe for help in communicating with authors during initiation and completion of the Research Topic. All authors in this Research Topic have provided their broad perspectives on transcriptional regulation, whose insightful thoughts will both benefit the field and be appreciated by fellow researchers. We are indebted to our reviewers/review editors for their contribution to this work.

9. Qin X, Xu H, Gong W, Deng W. The tumor cytosol miRNAs, fluid miRNAs, and exosome miRNAs in lung cancer. Front Oncol (2014) 6:1-7. doi:10.3389/ fonc. 2014.00357

10. Oltra E. Relevance of splicing on tumor-released exosome landscape: implications in cancer therapeutics. Front Endocrinol (2014) 5:1-3. doi:10.3389/fendo. 2014.00194

11. Davis PJ, Glinsky GV, Lin H-Y, Leith JT, Hercbergs A, Tang H-Y, et al. Cancer cell gene expression modulated from plasma membrane integrin $\alpha v \beta 3$ by thyroid hormone and nanoparticulate tetrac. Front Endocrinol (2015) 5:1-7. doi:10.3389/fendo.2014.00240

12. Davis PJ, Glinsky GV, Lin H-Y, Leith JT, Hercbergs A, Tang H-Y, et al Corrigendum: cancer cell gene expression modulated from plasma membrane integrin $\alpha v \beta 3$ by thyroid hormone and nanoparticulate tetrac. Front Endocrinol (2015) 6:1-1. doi:10.3389/fendo.2015.00098

Conflict of Interest Statement: The author declare that the research was conducted in the absent of any commercial or financial relationships that could be construed as a potential conflict of interest.

Copyright (c) 2015 Zhou. This is an open-access article distributed under the terms of the Creative Commons Attribution License (CC BY). The use, distribution or reproduction in other forums is permitted, provided the original author(s) or licensor are credited and that the original publication in this journal is cited, in accordance with accepted academic practice. No use, distribution or reproduction is permitted which does not comply with these terms. 